International Journal of Pure and Applied Mathematics

Volume 103 No. 2 2015, 263-279

ISSN: 1311-8080 (printed version); ISSN: 1314-3395 (on-line version)

url: http://www.ijpam.eu

doi: http://dx.doi.org/10.12732/ijpam.v103i2.12

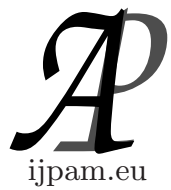

\title{
APPLICATION OF SHIFTED MÜNTZ-LEGENDRE POLYNOMIALS FOR SOLVING FRACTIONAL DIFFERENTIAL EQUATIONS
}

\author{
Mojtaba Rasouli Gandomani ${ }^{1}$, M. Tavassoli Kajani ${ }^{2} \S$ \\ 1,2 Department of Mathematics \\ Isfahan (Khorasgan) Branch \\ Islamic Azad University \\ Isfahan, IRAN
}

\begin{abstract}
In this paper, the technique of the interval division based on the shifted Müntz-Legendre polynomials is used to present an approximate solution for the fractional differential equations. The first step of proposed method is to divide the interval which is considered for the solution into several subintervals with a specific step size. We obtain an approximate solution of the problem on each of subintervals using the collocation method and the shifted MüntzLegendre polynomials. The use of the shifted Müntz- Legendre polynomials and the technique of the interval division simultaneously lead to more accuracy. The accuracy and convergence of the method are demonstrated with several numerical examples.
\end{abstract}

AMS Subject Classification: 34K28, 65R99, 65L99

Key Words: shifted Müntz-Legendre polynomials, fractional differential equations, approximate solution, collocation method

\section{Introduction}

Since 1695, the fractional computing and especially the fractional differential

Received: April 20, 2015

(C) 2015 Academic Publications, Ltd.

${ }^{\S}$ Correspondence author 
equations have attracted the interest of many researchers and mathematicians including pioneers such as Leibniz, Hopital, Euler and Laplace, etc. $[1,2,3,4]$ For a long time, the fractional computing was only considered by the pure mathematicians. Recently, it has many applications in different areas such as physics, chemistry, electronics, mechanics and even human sciences, etc. $[5,6$, $7,8,9,10,11]$ In this paper, we aim to find an approximate solution for the differential equations as follows:

$$
\left\{\begin{array}{l}
D_{*}^{\alpha} u(t)=g(t, u(t)), \quad 0<\alpha<1, \quad t \in[0, T] \\
u(0)=\mu,
\end{array}\right.
$$

where $D_{*}^{\alpha}$ denotes the fractional derivative of order $\alpha, g$ is a function of $t$ and $u(t)$; And $\mu$ is a constant number.

So far, various analytical and numerical methods have been proposed for solving these equations. For instance, Ford and Diethelm [12] have described the existence and uniqueness of the nonlinear fractional differential equations. Dehghan and Saadatmandi [13] have construced an operational matrix to solve these equations. Scherer et al. [14] have presented a numerical method to solve the fractional differential equations via the Grunwald-Letnikov definition of the fractional derivatives. Jafari [15] have applied Legendre wavelets to solve these equations. Esmaeili [16] et al. have made use of the collocation method and Muntz polynomials to solve them. In [17] an algorithm based on Adomian decomposition method is expressed to solve the fractional differential equations. Some of the analytical and numerical methods that have been used to solve the fractional differential equations can be found in $[18,19,20,21,22,23,24]$.

In this paper, we use the Caputo formula to obtain fractional derivative which is defined for $0<\alpha<1$ as follows:

$$
D_{*}^{\alpha} y(t)=\frac{1}{\Gamma(1-\alpha)} \int_{0}^{t}(t-\tau)^{-\alpha} y^{\prime}(\tau) d \tau,
$$

Also, we would employ the interval division and the shifted Müntz-Legendre polynomials methods which lead to get more accurate solutions.

The rest of paper is organized as follows: Section 2 devoted to some preliminaries. In this section we introduce Jacobi and Müntz-Legendre polynomials. The shifted Müntz-Legendre polynomials introduce in this section as well. The next section is devoted to presentation of the shifted Müntz-Legendre polynomials and a stable recursive relation for evaluating these functions then investigation of these polynomials' properties thereafter explanation of the proposed method. Finally, some examples are given to demonstrate the accuracy and efficiency of the proposed method. 


\section{Preliminaries}

\subsection{Jacobi Polynomials}

The Jacobi polynomials are orthogonal on the interval $[-1,1]$ with respect to the weight function $w^{(\alpha, \beta)}(x)=(1-x)^{\alpha}(1+x)^{\beta}$ where $\alpha, \beta>-1$. These polynomials can be obtained by the following relation:

$$
J_{k}^{(\alpha, \beta)}(x)=\sum_{m=0}^{k} \frac{(-1)^{k-m}(1+\beta)_{k}(1+\alpha+\beta)_{k+m}}{m !(k-m) !(1+\beta)_{m}(1+\alpha+\beta)_{k}}\left(\frac{1+x}{2}\right)^{m},
$$

where $(d)_{0}=1$ and $(d)_{k}=d(d+1) \cdots(d+k+1)$.

In addition, we have the following recursive relation for evaluating the Jacobi polynomials:

$$
\begin{aligned}
& J_{0}^{(\alpha, \beta)}(x)=1, \quad J_{1}^{(\alpha, \beta)}(x)=\frac{1}{2}((\alpha-\beta)+(\alpha+\beta+2) x), \\
& a_{k}^{(\alpha, \beta)} J_{k+1}^{(\alpha, \beta)}(x)=b_{k}^{(\alpha, \beta)}(x) J_{k}^{(\alpha, \beta)}(x)-c_{k}^{(\alpha, \beta)} J_{k-1}^{(\alpha, \beta)}(x), \\
& \quad a_{k}^{(\alpha, \beta)}=2(k+1)(k+\alpha+\beta+1)(2 k+\alpha+\beta), \\
& \quad b_{k}^{(\alpha, \beta)}(x)=(2 k+\alpha+\beta+1)\left((2 k+\alpha+\beta)(2 k+\alpha+\beta+2) x+\alpha^{2}-\beta^{2}\right), \\
& \quad c_{k}^{(\alpha, \beta)}=2(k+\alpha)(k+\beta)(2 k+\alpha+\beta+2) .
\end{aligned}
$$

As well as, the recursive relation for getting the initial derivative of the Jacobi polynomials is as follows:

$$
\frac{d}{d x} J_{k}^{(\alpha, \beta)}(x)=\frac{1}{2}(k+\alpha+\beta+1) J_{k-1}^{(\alpha+1, \beta+1)}(x)
$$

\subsection{Müntz-Legendre Polynomials}

Let $\Lambda_{n}=\left\{\lambda_{1}, \lambda_{2}, \ldots, \lambda_{n}\right\}$ be a complex sequence such that $\operatorname{Re}\left(\lambda_{k}\right)>-\frac{1}{2}, k=$ $1,2, \ldots, n$ and $\Gamma$ is a simple curve surrounding all the zeros of the denominator in the following function

$$
w_{n}(t)=\prod_{k=0}^{n-1} \frac{t-\bar{\lambda}_{k}+1}{t-\lambda_{k}} \cdot \frac{1}{t-\lambda_{n}}
$$


In this case, the Müntz-Legendre polynomials are defined as follows [25, 26]:

$$
L\left(\Lambda_{n}, x\right)=\frac{1}{2 \pi i} \int_{\Gamma} w_{n}(t) x^{t} d t
$$

Also, if $\lambda_{k} \neq \lambda_{j}$ be true for $k \neq j$, then according to the above definition and Cauchy residue theorem, the Müntz-Legendre polynomials can be defined as follows:

$$
L_{n}(x)=L\left(\Lambda_{n}, x\right)=\sum_{k=0}^{n} C_{n, k} x^{\lambda_{n}}, \quad C_{n, k}=\frac{\prod_{v=0}^{n-1}\left(\lambda_{k}+\bar{\lambda}_{v}+1\right)}{\prod_{v=0, v \neq k}^{n}\left(\lambda_{k}-\lambda_{v}\right)}
$$

These polynomials are orthogonal on the interval $(0,1]$ with respect to the weight function $w(t)=1$ and the following relation hold:

$$
\left(L_{n}, L_{m}\right)=\int_{0}^{1} L_{n}(x) L_{m}(x) d x=\frac{\delta_{m n}}{\lambda_{n}+\bar{\lambda}_{n}+1}
$$

In which $\delta_{m n}$ denotes the Kronecker delta.

If all $\lambda_{k}$ are chosen so that $\lambda_{k}=\alpha k$, the Müntz-Legendre polynomials on the interval $[0, T)$ are defined as follows ( $\alpha$ is a positive real number):

$$
L_{n}(t: \alpha)=\sum_{k=0}^{n} C_{n, k}\left(\frac{t}{T}\right)^{\alpha k}, \quad C_{n, k}=\frac{(-1)^{n-k}}{\alpha^{n} k !(n-k) !} \prod_{v=0}^{n-1}((k+v) \alpha+1)
$$

\subsection{Shifted Müntz-Legendre Polynomials}

Assuming $I=\left[x_{i}, x_{i+1}\right]$ is an arbitrary interval with length $h$. The shifted Müntz-Legendre polynomials of order $n$ for parameter $\alpha$, on the interval $I=$ $\left[x_{i}, x_{i+1}\right]$ are defined as follows:

$$
l_{I, n}(t: \alpha)=\sum_{k=0}^{n} C_{n, k}\left(\frac{t-x_{i}}{h}\right)^{\alpha k}, \quad x_{i} \leq t \leq x_{i+1}
$$

where $C_{n, k}$ is defined in Eq. (4). 


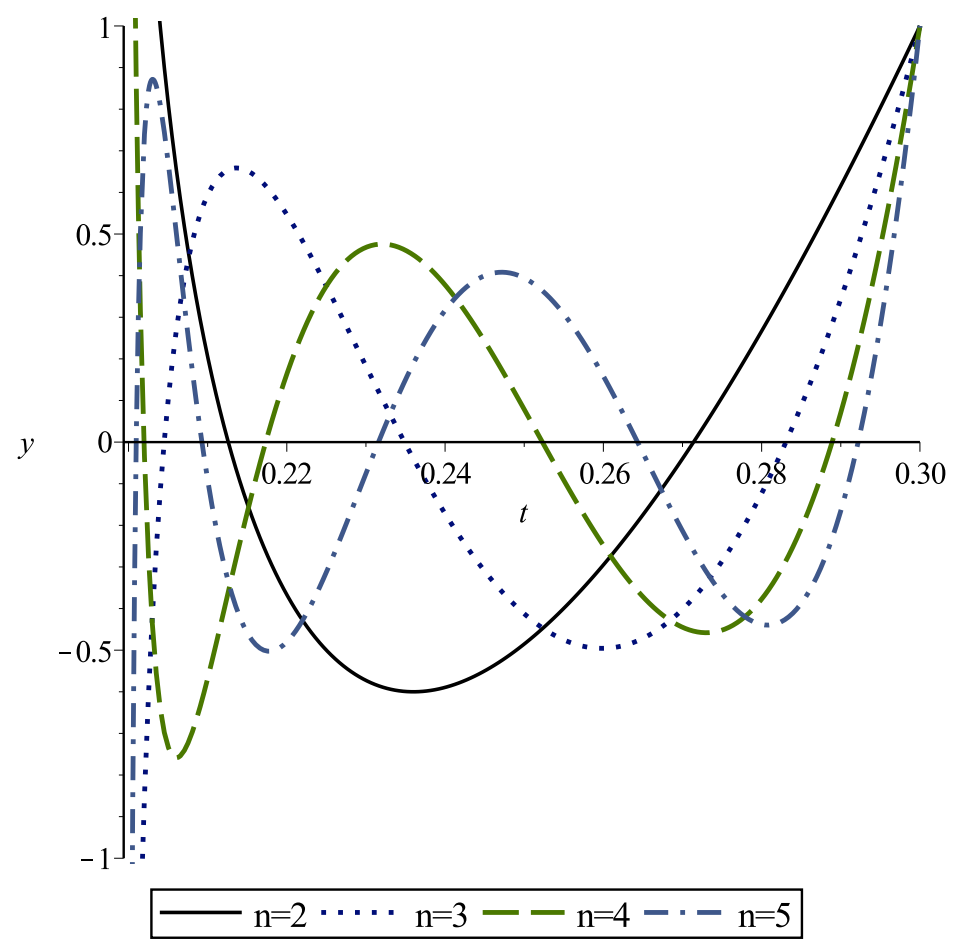

Figure 1: $l_{I, n}(t: \alpha)$ for $I=[0.2,0.3], \alpha=0.5$ and $n=2,3,4,5$.

\section{Shifted Müntz-Legendre Polynomials' Properties}

These functions are orthogonal on the interval $I$ with respect to the weight function $w(t)=1$ and the following hold:

$$
\begin{aligned}
& \int_{x_{i}}^{x_{i+1}} l_{I, n}(x: \alpha) l_{I, m}(x: \alpha) d x=\frac{h \delta_{n, m}}{1+2 \alpha n}, \\
& l_{I, n}\left(x_{i+1}: \alpha\right)=1,
\end{aligned}
$$

where $l_{I, n}(x: \alpha)$ has exactly $n$ distinct zeros on the interval $\left[x_{i}, x_{i+1}\right]$.

The Fig. 1 shows $l_{I, n}(t: \alpha)$ for $I=[0.2,0.3], \alpha=0.5$ and $n=2,3,4,5$.

The sum of the coefficients of these polynomials is always equal to 1. As Milovanovic said the higher $n$ the higher coefficient of polynomials [27]. It leads to 
increase the computing error. That's why we offer a stable method to calculate these polynomials.

Theorem 1. Let $\alpha>0$ be a real number and $h=x_{i+1}-x_{i}, x \in\left[x_{i}, x_{i+1}\right]$, then

$$
l_{I, n}(x: \alpha)=J_{n}^{\left(0, \frac{1}{\alpha}-1\right)}\left(2\left(\frac{x-x_{i}}{h}\right)^{\alpha}-1\right)
$$

Proof. According to the proposed relation for the Jacobi polynomials, we have:

$$
\begin{aligned}
J_{n}^{\left(0, \frac{1}{\alpha}-1\right)}\left(2\left(\frac{x-x_{i}}{h}\right)^{\alpha}-1\right) & =\sum_{k=0}^{n} \frac{(-1)^{n-k}\left(\frac{1}{\alpha}\right)_{n}\left(\frac{1}{\alpha}\right)_{n+k}}{k !(n-k) !\left(\frac{1}{\alpha}\right)_{n}\left(\frac{1}{\alpha}\right)_{k}}\left(\frac{x-x_{i}}{h}\right)^{k \alpha} \\
& =\sum_{k=0}^{n} \frac{(-1)^{n-k}\left(\frac{1}{\alpha}\right)_{n+k}}{k !(n-k) !\left(\frac{1}{\alpha}\right)_{k}}\left(\frac{x-x_{i}}{h}\right)^{k \alpha} \\
& =\sum_{k=0}^{n} \frac{(-1)^{n-k}}{\alpha^{n} k !(n-k) !} \prod_{v=0}^{n-1}((k+v) \alpha+1)\left(\frac{x-x_{i}}{h}\right)^{k \alpha} \\
& =\sum_{k=0}^{n} C_{n, k}\left(\frac{x-x_{i}}{h}\right)^{k \alpha} \\
& =l_{I, n}(x: \alpha)
\end{aligned}
$$

In addition, in the view of the proposed recursive formula for the Jacobi polynomials, the shifted Müntz-Legendre polynomials can be obtained by using the recursive formula:

$$
\begin{aligned}
& l_{I, 0}(x: \alpha)=1, \quad l_{I, 1}(x: \alpha)=\left(\frac{1}{\alpha}+1\right)\left(\frac{x-x_{i}}{h}\right)^{\alpha}-\frac{1}{\alpha}, \\
& a_{I, n} l_{I, n+1}(x: \alpha)=b_{I, n}(x) l_{I, n}(x: \alpha)-c_{I, n} l_{I, n-1}(x: \alpha), \\
& a_{I, n}=a_{n}^{\left(0, \frac{1}{\alpha}-1\right)}, \quad b_{I, n}(x)=b_{n}^{\left(0, \frac{1}{\alpha}-1\right)}\left(2\left(\frac{x-x_{i}}{h}\right)^{\alpha}-1\right), \quad c_{I, n}=c_{n}^{\left(0, \frac{1}{\alpha}-1\right)}
\end{aligned}
$$

Obtaining the shifted Müntz-Legendre polynomials via the above recursive formula leads to more stability. Since $l_{I, n}\left(x_{i+1}: \alpha\right)=1$, so absolute error 


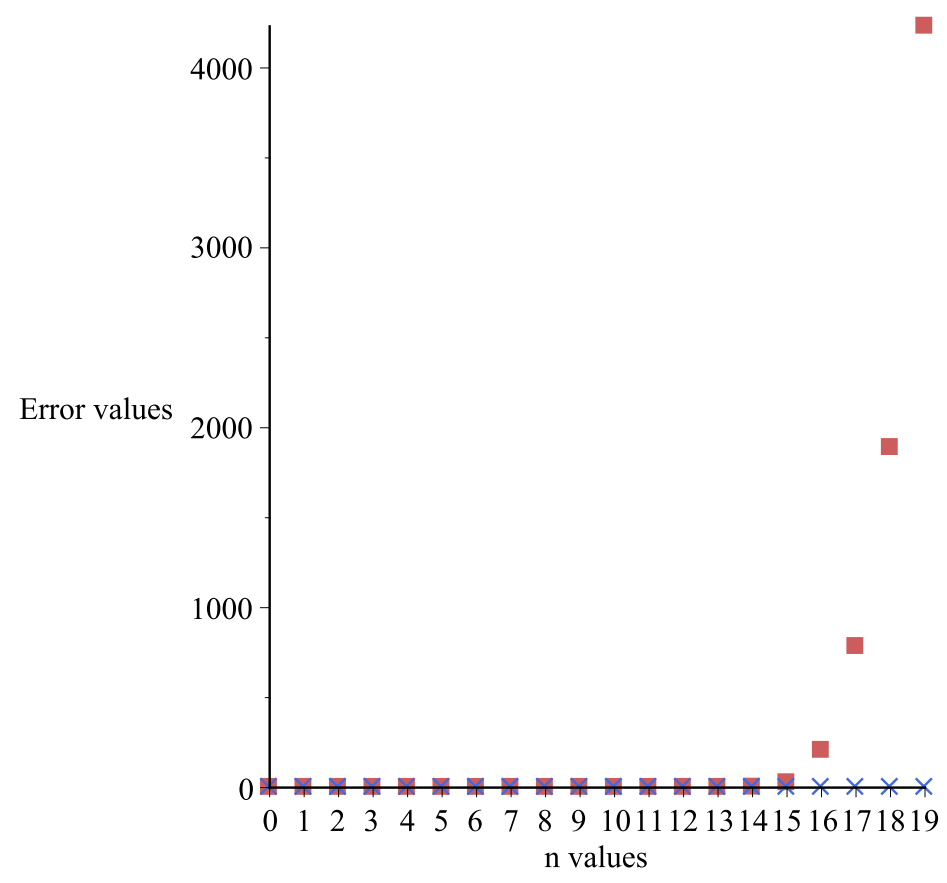

\begin{tabular}{|lll}
\hline By using(5) $\quad \times \quad$ By using(7) \\
\hline
\end{tabular}

Figure 2: Error values for $l_{I, n}(0.3: 0.5), I=[0.2,0.3]$.

obtained for $l_{I, n}\left(x_{i+1}: \alpha\right)$ using Eqs. (5) and (7) on $I=[0.2,0.3], \alpha=0.5$ for different $n$ is compered in Fig. 2.

Fig. 2 depicts that for all $n>15$ the error obtained for Eq. (5) becomes high, whereas the error obtained for the Eq. (7) shows that it has a good stability.

Also, in the view of Eq. (2) derivative of the Jacobi polynomials, $l_{I, n}^{\prime}(x: \alpha)$ can be obtained as follows:

$$
\frac{d}{d x} l_{I, n}(x: \alpha)=\frac{1}{h^{\alpha}}(\alpha n+1)\left(x-x_{i}\right)^{\alpha-1} J_{n-1}^{\left(1, \frac{1}{\alpha}\right)}\left(2\left(\frac{x-x_{i}}{h}\right)^{\alpha}-1\right) .
$$

\subsection{Approximate of the Functions}

According to what has been already mentioned, we get an approximate of a given function $f(x)$ on the interval $I=\left[x_{i}, x_{i+1}\right]$ in terms of the linear combi- 
nation of the shifted Müntz-Legendre functions on the interval $I$ :

$$
f(x) \approx \sum_{k=0}^{N} c_{k} l_{I, k}(x: \alpha)=C^{T} L_{I}
$$

where

$$
C=\left[c_{0}, c_{1}, \ldots, c_{N}\right]^{T} \quad \text { and } \quad L_{I}=\left[l_{I, 0}(x: \alpha), l_{I, 1}(x: \alpha), \ldots, l_{I, N}(x: \alpha)\right]^{T}
$$

According to the Eq. (6), the unknown coefficients $c_{k}$ can be therefore obtained for $k=0,1, \ldots, N$ as follows:

$$
c_{k}=\frac{2 k \alpha+1}{x_{i+1}-x_{i}} \int_{x_{i}}^{x_{i+1}} f(x) l_{I, k}(x: \alpha) d x
$$

\section{Solving of the Fractional Differential Equation}

For solving fractional differential equation, we should initially divide the interval $[0, T]$ to $M$ subintervals $I_{k}=[k h,(k+1) h]$ for $k=0,1, \ldots, M-1$. Let $u_{k}$ be an approximate solution of the function on the subinterval $I_{k}$ such that the Eq. (1) on the subinterval $I_{k}$ is:

$$
\left\{\begin{array}{l}
D_{*}^{\alpha} u_{k}(t)=g\left(t, u_{k}(t)\right), \quad 0<\alpha<1, \quad t \in[k h,(k+1) h] \\
u_{k}(k h)=y_{k},
\end{array}\right.
$$

in which:

$$
y_{k}= \begin{cases}\mu, & k=0 \\ u_{k-1}(k h), & k=1,2, \ldots, M-1,\end{cases}
$$

The initial value for the first subinterval is the same as the initial value given in the Eq. (1) and for the subintervals $I_{k}, k=1,2, \ldots, M-1$, the initial value is obtained by using approximate solution on the subinterval $I_{k-1}$.

Now, according to what has been already mentioned the approximate solution for the problem can be obtained by using the shifted Müntz-Legendre functions for any subinterval as follows:

$$
u_{k}(t) \approx C_{k}^{T} L_{I_{k}}, \quad k=0,1, \ldots, M-1 .
$$

In this case, the approximate solution of the problem on $[0, T]$ can be obtained by calculating $u_{0}, u_{1}, \ldots, u_{M-1}$. 
The equation for the $k$ th subinterval, according to the Caputo form for fractional derivative, is turned into:

$$
\begin{array}{r}
\frac{1}{\Gamma(1-\alpha)}\left(\sum_{s=0}^{k-1} \int_{s h}^{(s+1) h} \frac{u_{s}^{\prime}(x) d x}{(1-x)^{\alpha}}+\int_{k h}^{t} \frac{C_{k}^{T} L_{I_{k}}^{\prime}(x) d x}{(t-x)^{\alpha}}\right)=g\left(t, C_{k}^{T} L_{I_{k}}(t)\right) \\
t \in I_{k}
\end{array}
$$

With initial condition:

$$
C_{k}^{T} L_{I_{k}}(k h)=y_{k}
$$

in which:

$$
L_{I_{k}}^{\prime}(x)=\left[l_{I_{k}, 0}^{\prime}(x: \alpha), l_{I_{k}, 1}^{\prime}(x: \alpha), \ldots, l_{I_{k}, N}^{\prime}(x: \alpha)\right]^{T}
$$

By substituting the points' $\theta_{k, i},(i=1,2, \ldots, N)$, the Chebyshev's point, in Eq. (9), we have:

$$
\begin{aligned}
\frac{1}{\Gamma(1-\alpha)}\left(\sum_{s=0}^{k-1} \int_{s h}^{(s+1) h} \frac{u_{s}^{\prime}(x) d x}{(1-x)^{\alpha}}+\int_{k h}^{\theta_{k, i}} \frac{C_{k}^{T} L_{I_{k}}^{\prime}(x) d x}{\left(\theta_{k, i}-x\right)^{\alpha}}\right) & =g\left(\theta_{k, i}, C_{k}^{T} L_{I_{k}}\left(\theta_{k, i}\right)\right) \\
i & =1,2, \ldots, N
\end{aligned}
$$

where $i=1,2, \ldots, N$ and all of $\theta_{k, i}$ are obtained by the following relation:

$$
\theta_{k, i}=\frac{(2 k+1) h}{2}-\frac{h}{2} \cos \left(\frac{\pi i}{N}\right), \quad i=1,2, \ldots, N .
$$

Now, the Eqs. (10) and (11) are formed an algebraic equations system with $N+1$ equations and $N+1$ unknown parameters. The unknown coefficients $C_{k}^{T}$ are obtained by solving such equations system. Then we can obtain $u_{k}(t)$, an approximate of the equation solution on the interval $I_{k}$, by replacing these coefficients into Eq. (8).

\section{Illustrative Examples}

In this section, we apply the proposed method to some numerical examples.

Example 1. Consider the following fractional differential equation [13, $16]$ :

$$
D_{*}^{\alpha} u(t)=\frac{40320}{\Gamma(9-\alpha)} t^{8-\alpha}-\frac{3 \Gamma\left(5+\frac{\alpha}{2}\right)}{\Gamma\left(5-\frac{\alpha}{2}\right)} t^{4-\frac{\alpha}{2}}+\frac{9}{4} \Gamma(\alpha+1)+\left(\frac{3}{2} t^{\frac{\alpha}{2}}-t^{4}\right)^{3}-(u(t))^{\frac{3}{2}},
$$


Table 1: Absolute error for $N=10, h=0.1$, Ex. 1

\begin{tabular}{|c|c|c|c|c|c|c|c|c|}
\hline \multirow[b]{3}{*}{$x$} & \multicolumn{2}{|c|}{$\alpha=0.2$} & \multicolumn{2}{|c|}{$\alpha=0.4$} & \multicolumn{2}{|c|}{$\alpha=0.6$} & \multicolumn{2}{|c|}{$\alpha=0.8$} \\
\hline & Present & Method & Present & Method & Present & Method & Present & Method \\
\hline & Method & {$[13]$} & Method & [13] & Method & [13] & Method & {$[13]$} \\
\hline 0.1 & $3.4 e-6$ & $2.2 e-1$ & $9.5 e-11$ & $6.3 e-2$ & $3.2 e-12$ & $1.5 e-2$ & $3.1 e-7$ & $2.9 e-3$ \\
\hline 0.3 & $2.3 e-5$ & $2.3 e-1$ & $3.4 e-9$ & $6.0 e-2$ & $2.8 e-9$ & $1.3 e-2$ & $7.7 e-6$ & $2.1 e-3$ \\
\hline 0.5 & $4.4 e-5$ & $3.6 e-2$ & $4.2 e-9$ & $2.4 e-2$ & $6.1 e-9$ & $9.6 e-3$ & $4.9 e-6$ & $2.3 e-3$ \\
\hline 0.7 & $2.6 e-5$ & $5.3 e-1$ & $9.7 e-9$ & $1.2 e-1$ & $3.7 e-9$ & $2.1 e-2$ & $3.0 e-6$ & $2.5 e-3$ \\
\hline 0.9 & $1.7 e-4$ & $1.7 e-0$ & $5.3 e-9$ & $3.0 e-1$ & $4.9 e-9$ & $3.7 e-2$ & $1.9 e-7$ & $2.1 e-3$ \\
\hline
\end{tabular}

Table 2: Maximum absolute error for $h=0.1$, Ex. 1 .

\begin{tabular}{clllllll}
\hline $\mathrm{N}$ & $\alpha=0.25$ & $\alpha=0.5$ & $\alpha=0.75$ & $\alpha=0.8$ & $\alpha=0.85$ & $\alpha=0.9$ & $\alpha=0.95$ \\
\hline 5 & $1.8 e-3$ & $1.0 e-3$ & $8.9 e-5$ & $8.9 e-4$ & $2.6 e-4$ & $2.9 e-5$ & $7.2 e-4$ \\
10 & $7.2 e-5$ & $7.5 e-7$ & $3.6 e-8$ & $3.1 e-7$ & $6.3 e-6$ & $3.3 e-7$ & $9.3 e-7$ \\
15 & $4.0 e-8$ & $6.5 e-10$ & $1.2 e-9$ & $4.6 e-9$ & $3.7 e-10$ & $7.1 e-10$ & $1.6 e-10$ \\
20 & $2.2 e-10$ & $3.8 e-11$ & $5.2 e-11$ & $2.9 e-10$ & $1.5 e-11$ & $8.8 e-11$ & $6.7 e-11$ \\
25 & $1.0 e-11$ & $2.6 e-13$ & $4.0 e-13$ & $3.9 e-13$ & $6.6 e-12$ & $2.0 e-13$ & $3.8 e-14$ \\
30 & $8.8 e-14$ & $9.6 e-16$ & $3.2 e-15$ & $9.8 e-16$ & $7.8 e-16$ & $6.0 e-16$ & $3.1 e-15$ \\
35 & $3.3 e-17$ & $8.4 e-18$ & $1.1 e-17$ & $2.3 e-18$ & $9.6 e-19$ & $5.4 e-19$ & $6.1 e-18$ \\
\hline
\end{tabular}

in which $0<\alpha<1$ and the initial value is $y(0)=0$. Thus the exact solution of the equation is:

$$
y(t)=t^{8}-3 t^{4+\frac{\alpha}{2}}+\frac{9}{4} t^{\alpha} .
$$

Table 1 depicts the absolute error obtained for different values of $x$ and $\alpha$. Also, Table 2 displays the maximum absolute error obtained for different values of $N$ and $\alpha$. In Fig. 3, the chart of the obtained solution has been shown for different values of $\alpha$ and $N=20, h=0.1$.

Example 2. Consider the fractional differential equation with the following initial value $[13,16]$ :

$$
\left\{\begin{array}{l}
D_{*}^{\alpha} u(t)+u(t)=0, \quad t \in[0,1], \quad 0<\alpha \leq 1, \\
u(0)=0,
\end{array}\right.
$$

The exact solution of the equation is

$$
u(x)=\sum_{k=0}^{\infty} \frac{\left(-x^{\alpha}\right)^{k}}{\Gamma(k \alpha+1)}, \quad 0<\alpha \leq 1
$$

Table 3 depicts the absolute error obtained for different values of $x$ and $\alpha$. Also, Table 4 displays the maximum absolute error obtained for different values of $N$ 


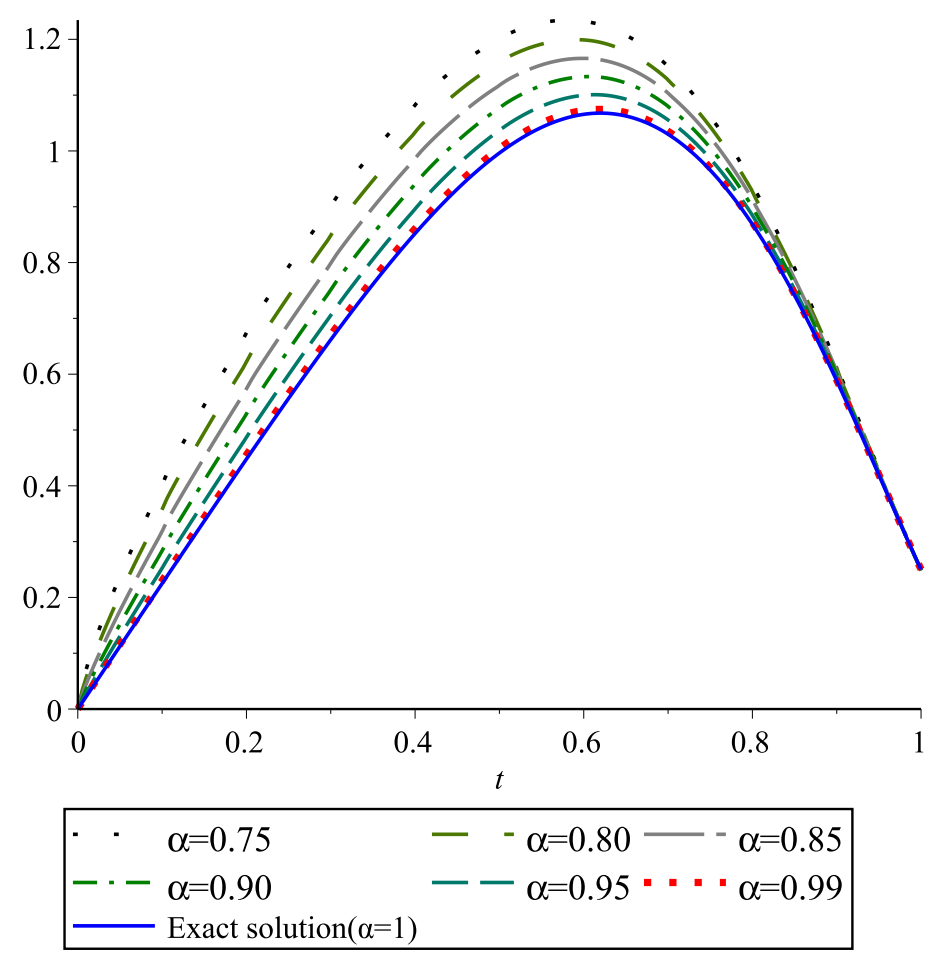

Figure 3: Numerical solution for $N=20, h=0.1$ and different values of $\alpha$, Ex. 1.

and $\alpha$. In Fig. 4 , the chart of the obtained solution has been shown for different values of $\alpha$ and $N=20, h=0.1$.

Example 3. Consider the fractional differential equation:

$$
\left\{\begin{array}{l}
D_{*}^{\alpha} u(t)=\frac{\Gamma(3)}{\Gamma(3-\alpha)} t^{2-\alpha}, \quad t \in[0,2], \quad 0<\alpha \leq 1, \\
u(0)=1,
\end{array}\right.
$$

The exact solution of the equation is

$$
u(x)=t^{2}+1
$$

In Fig. 5 the absolute error has been shown for $\alpha=0.5, N=5$ and different values of $h$ on the interval $[0,2]$. 
Table 3: Absolute error for different values of $\alpha$ and $N=10, h=0.1$, Ex. 2

\begin{tabular}{|c|c|c|c|c|c|c|c|c|}
\hline \multirow[b]{2}{*}{$x$} & \multicolumn{2}{|c|}{$\alpha=0.2$} & \multicolumn{2}{|c|}{$\alpha=0.4$} & \multicolumn{2}{|c|}{$\alpha=0.6$} & \multicolumn{2}{|c|}{$\alpha=0.8$} \\
\hline & $\begin{array}{l}\text { Present } \\
\text { Method }\end{array}$ & $\begin{array}{l}\text { Method } \\
{[13]}\end{array}$ & $\begin{array}{l}\text { Present } \\
\text { Method }\end{array}$ & $\begin{array}{l}\text { Method } \\
{[13]}\end{array}$ & $\begin{array}{l}\text { Present } \\
\text { Method }\end{array}$ & $\begin{array}{l}\text { Method } \\
{[13]}\end{array}$ & $\begin{array}{l}\text { Present } \\
\text { Method }\end{array}$ & $\begin{array}{l}\text { Methoc } \\
{[13]}\end{array}$ \\
\hline 0.1 & $7.0 e-11$ & $2.9 e-1$ & $2.1 e-12$ & $3.9 e-1$ & $1.5 e-12$ & $6.7 e-3$ & $1.2 e-7$ & $1.1 e-3$ \\
\hline 0.3 & $6.3 e-9$ & $4.5 e-1$ & $4.4 e-10$ & $5.1 e-1$ & $2.0 e-10$ & $2.0 e-5$ & $1.7 e-6$ & $2.1 e-4$ \\
\hline 0.5 & $9.3 e-9$ & $7.4 e-1$ & $6.6 e-10$ & $7.3 e-1$ & $2.2 e-10$ & $5.2 e-3$ & $9.8 e-6$ & $8.4 e-4$ \\
\hline 0.7 & $8.0 e-10$ & $3.7 e-1$ & $5.7 e-9$ & $3.3 e-1$ & $3.1 e-10$ & $4.4 e-3$ & $6.2 e-6$ & $8.7 e-4$ \\
\hline 0.9 & $8.8 e-10$ & $2.0 e-1$ & $4.4 e-9$ & $2.2 e-1$ & $3.1 e-10$ & $4.6 e-3$ & $6.9 e-5$ & $5.8 e-4$ \\
\hline
\end{tabular}

Table 4: Maximum absolute error for $h=0.1$, Ex. 2.

\begin{tabular}{clllllll}
\hline $\mathrm{N}$ & $\alpha=0.25$ & $\alpha=0.5$ & $\alpha=0.75$ & $\alpha=0.8$ & $\alpha=0.85$ & $\alpha=0.9$ & $\alpha=0.95$ \\
\hline 5 & $1.9 e-3$ & $1.0 e-4$ & $6.7 e-4$ & $8.1 e-4$ & $2.5 e-4$ & $1.5 e-2$ & $4.2 e-3$ \\
10 & $7.1 e-5$ & $3.7 e-7$ & $2.4 e-8$ & $2.1 e-7$ & $6.3 e-6$ & $3.3 e-7$ & $3.0 e-7$ \\
15 & $6.1 e-8$ & $3.5 e-10$ & $3.1 e-9$ & $7.6 e-9$ & $5.8 e-10$ & $6.0 e-10$ & $8.5 e-10$ \\
20 & $1.3 e-10$ & $1.7 e-11$ & $4.0 e-11$ & $2.2 e-10$ & $4.1 e-11$ & $3.8 e-11$ & $4.7 e-11$ \\
25 & $1.1 e-11$ & $3.4 e-13$ & $3.1 e-13$ & $2.2 e-13$ & $5.4 e-12$ & $2.3 e-13$ & $2.6 e-14$ \\
30 & $7.8 e-13$ & $1.5 e-15$ & $4.4 e-15$ & $1.8 e-16$ & $3.1 e-15$ & $2.0 e-15$ & $1.1 e-15$ \\
35 & $2.0 e-18$ & $3.9 e-19$ & $1.1 e-18$ & $5.3 e-19$ & $6.1 e-19$ & $1.0 e-19$ & $6.0 e-18$ \\
\hline
\end{tabular}

Example 4. Consider the Riccati equation in the following form [16]:

$$
\left\{\begin{array}{l}
D_{*}^{\alpha} u(t)=1+2 u(t)-(u(t))^{2}, \quad t \in[0,2], \quad 0<\alpha \leq 1, \\
u(0)=0,
\end{array}\right.
$$

The exact solution of the equation for $\alpha=1$ is as follows:

$$
u(t)=1+\sqrt{2} \tanh \left(\sqrt{2} t+\frac{1}{2} \ln \left(\frac{\sqrt{2}-1}{\sqrt{2}+1}\right)\right) .
$$

In Fig. 6, the chart of the obtained solution has been shown for different values of $\alpha$ and $N=20, h=0.1$.

\section{Conclusion}

In this paper, we introduced a numerical method for solving the fractional differential equations using the shifted Müntz-Legendre polynomials and the 


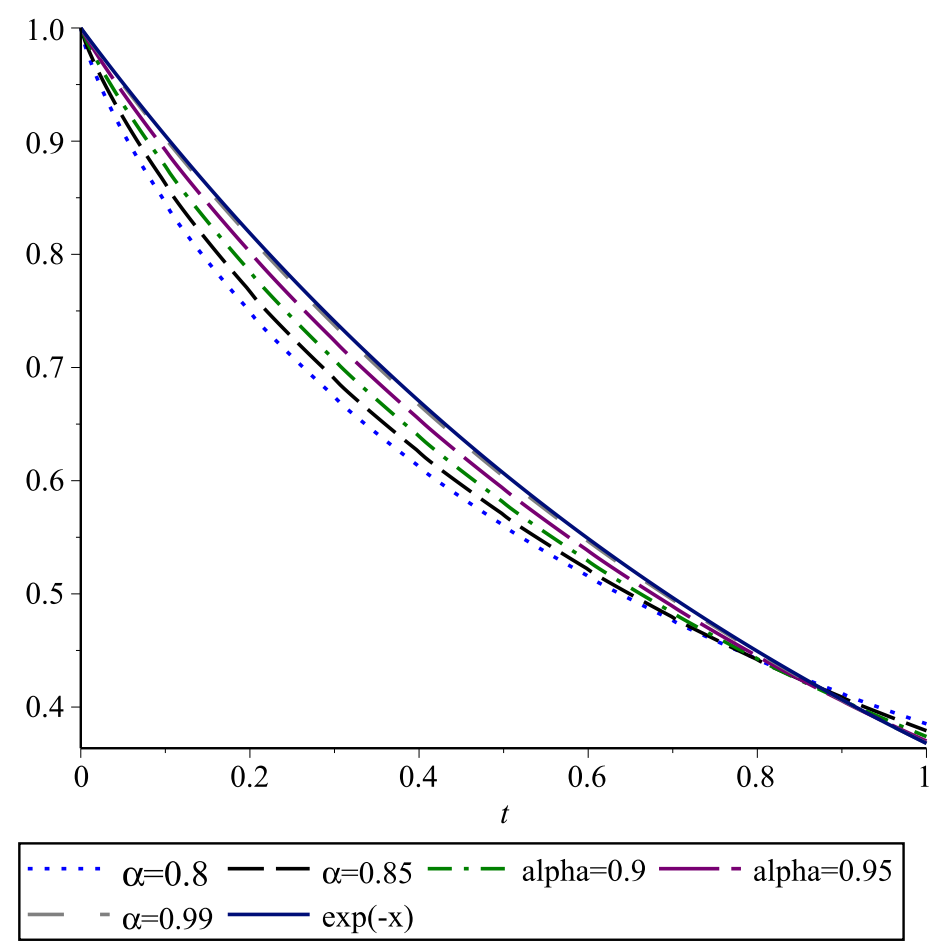

Figure 4: Numerical solution for $N=20, h=0.1$ and different values of $\alpha$, Ex. 2.

technique of the interval division. We presented a stable recursive formula for getting the shifted Müntz-Legendre polynomials by means of the recursive property of the Jacobi polynomials. Illustrative examples were included to demonstrate the accuracy and efficiency of the method. The result clearly showed that the presented method has very good accuracy and efficiency even with using few collocation points.

\section{References}

[1] B. Ross, The development of fractional calculus 1695-1900, Hist. Math. 4(1) (1977) 75-89.

[2] K.B. Oldham, J. Spanier, The Fractional Calculus, Academic Press, New York, 1974. 


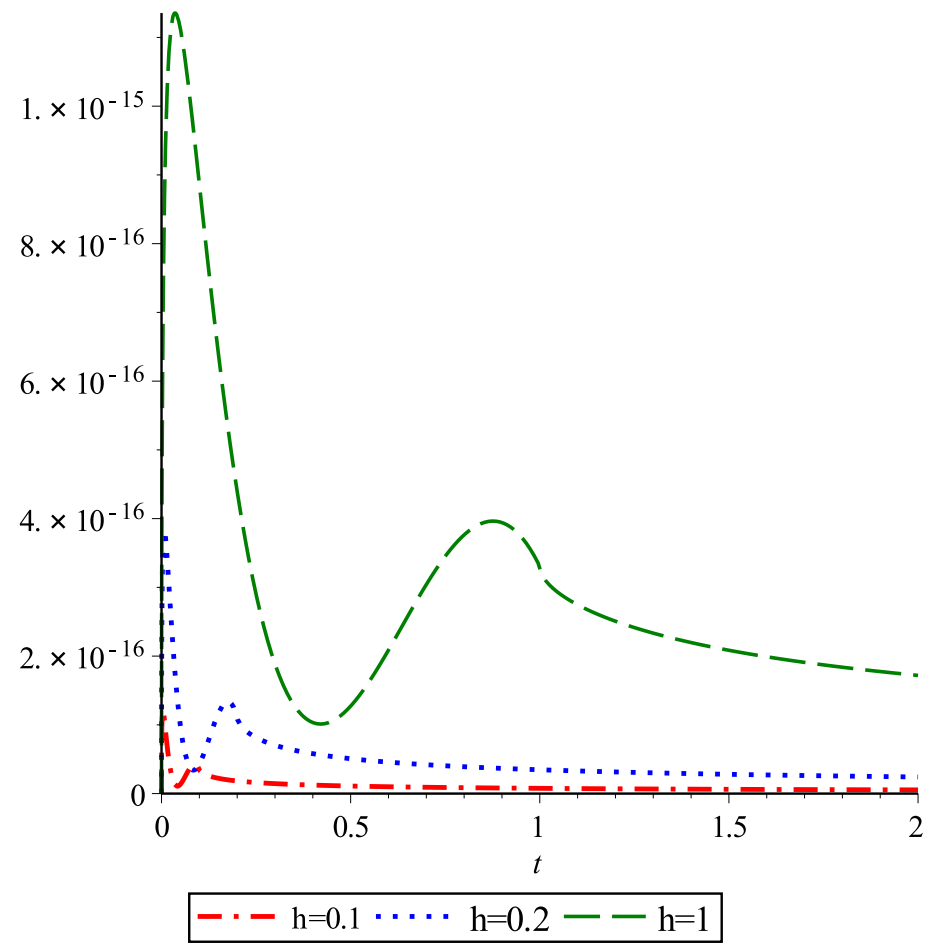

Figure 5: Absolute error for $\alpha=0.5, N=5$ and different values of $h$, Ex. 3.

[3] K.S. Miller, B. Ross, An Introduction to The Fractional Calculus and Fractional Differential Equations, Wiley, New York, 1993.

[4] J.T. Machado, V. Kiryakova, F. Mainardi, Recent history of fractional calculus, Commun. Nonlinear Sci. Numer. Simulat, 16 (2011) 1140-1153.

[5] R.L. Bagley, P.J. Torvik, A theoretical basis for the application of fractional calculus to viscoelasticity, J. Rheology, 27(3) (1983) 201-210.

[6] Y. Luchko, M. Rivero, J.J. Trujillo, M.P. Velasco, Fractional models, nonlocality, and complex systems, Comput. Math. Appl., 59(3) (2010) 10481056.

[7] Y.A. Rossikhin, M.V. Shitikova, Applications of fractional calculus to dynamic problems of linear and nonlinear hereditary mechanics of solids, Appl. Mech. Rev., 50 (1997) 15-67. 


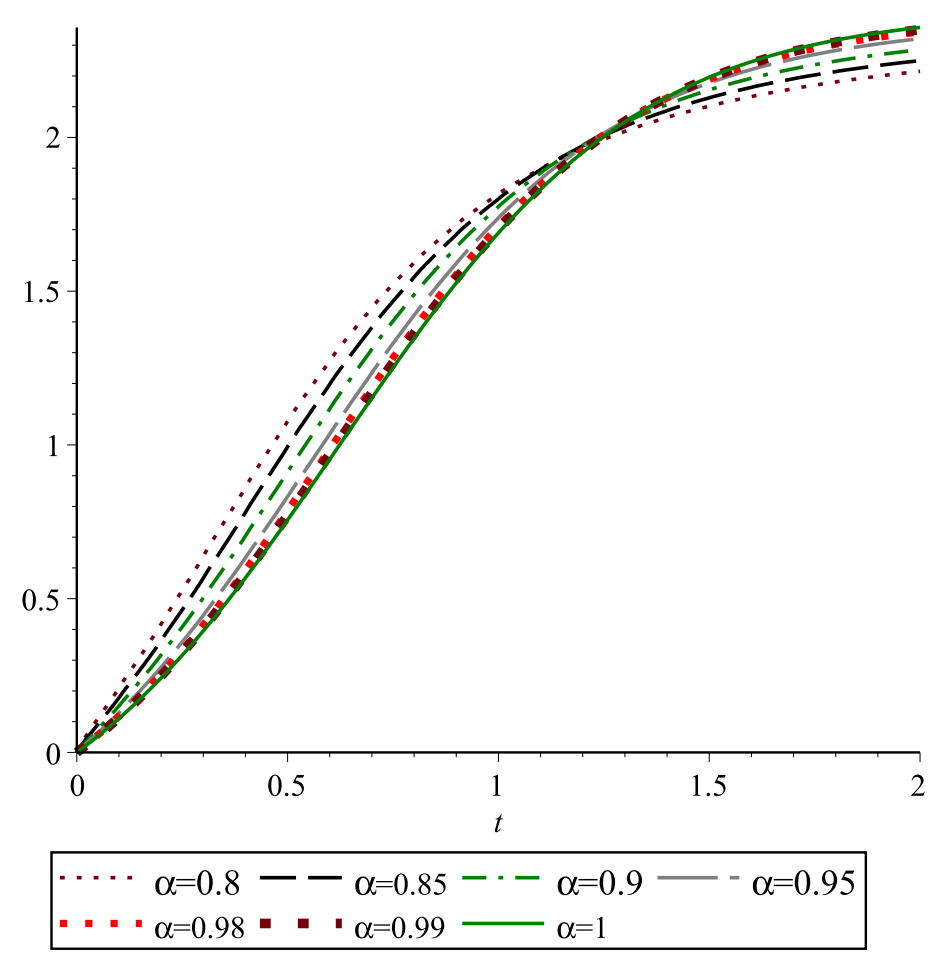

Figure 6: Numerical solution for $N=20, h=0.1$ and different values of $\alpha$, Ex. 4.

[8] L. Gaul, P. Klein, S. Kempfle, Impulse response function of an oscillator with fractional derivative in damping description, Mech. Res. Commun., 16(5) (1989) 297-305.

[9] T.S. Chow, Fractional dynamics of interfaces between soft-nanoparticles and rough substrates, Phys. Lett. A., 342 (2005) 148-155.

[10] L. Debnath, Recent applications of fractional calculus to science and engineering, Int. J. Math. Sci., 54 (2003) 3413-3442.

[11] G.M. Zaslavsky, Chaos, fractional kinetics, and anomalous transport, Phys. Rep., 371 (2002) 461-580.

[12] K. Diethelm, N.J. Ford, Analysis of Fractional Differential Equations, J. Math. Analy. Appl., 265 (2), (2002) 229248. 
[13] A. Saadatmandi, M. Dehghan, A new operational matrix for solving fractional-order differential equations, Comput. Math. Appl., 59(3) (2010) 1326-1336.

[14] R. Scherer, Shyam L. Kalla, Y. Tang, J. Huang, The Grnwald-Letnikov method for fractional differential equations, Comput. Math. Appl., 62 (2011) 902-917.

[15] H. Jafari , S.A. Yousefi , M.A. Firoozjaee , S. Momanic, C.M. Khalique, Application of Legendre wavelets for solving fractional differential equations, Comput. Math. Appl., 62 (2011) 1038-1045.

[16] S. Esmaeili, M. Shamsi, Y . Luchko, Numerical solution of fractional differential equations with a collocation method based on Müntz polynomials, Comput. Math. Appl., 62 (2011) 918-929.

[17] C. Li, Y. Wang, Numerical algorithm based on Adomian decomposition for fractional differential equations, Comput. Math. Appl., 57(10) (2009) 1672-1681.

[18] S. Momani, An explicit and numerical solutions of the fractional KdV equation, Math. Comput. Simulation, 70(2) (2005) 110-118.

[19] K. Diethelm, J. M. Ford, N. J. Ford, M. Weilbeer, Pitfalls in fast numerical solvers for fractional differential equations, Comput. Appl. Math., 186, (2006) 482-503.

[20] K. Diethelm, N. J. Ford, A.D. Freed, Y . Luchko, Algorithms for the fractional calculus: A selection of numerical methods, Comput. Methods Appl. Mech. Engrg., 194 (2005) 743-773.

[21] S. Esmaeili, M. Shamsi, A pseudo-spectral scheme for the approximate solution of a family of fractional differential equations, Commun. Nonlinear Sci. Numer. Simul., 16(9) (2011) 3646-3654.

[22] R. Garrappa, M. Popolizio, On accurate product integration rules for linear fractional differential equations, Comput. Appl. Math., 235 (2011) 10851097.

[23] F. Ghoreishi, S. Yazdani, An extension of the spectral Tau method for numerical solution of multi-order fractional differential equations with convergence analysis, Computers and Mathematics with Applications, 61 (2011) $30-43$. 
[24] S. Momani, Z. Odibat, V. Erturk, Generalized differential transform method for solving a space and time-fractional diffusion-wave equation, Phys. Lett. A, 370(5-6) (2007) 379-387.

[25] P.C. McCarthy, J.E. Sayre, B.L.R. Shawyer, Generalized Legendre polynomials, J. Math. Anal. Appl., 177 (1993) 530-537.

[26] P. Borwein, T. Erdélyi, J. Zhang, Müntz systems and orthogonal MüntzLegendre polynomials, Trans. Amer. Math. Soc., 342(2) (1994) 523-542.

[27] G.V. Milovanovic̀, Müntz orthogonal polynomials and their numerical evaluation, in: Applications and Computation of Orthogonal Polynomials, Ser. Numer. Math., vol. 131, Birkhuser, Basel, (1999) 179-194. 
